\title{
IMAGE SEGMENTATION BASED ON COLOR
}

\author{
Navkirat Kaur ${ }^{1}$, V. K. Banga ${ }^{2}$, Avneet Kaur ${ }^{3}$ \\ ${ }^{1,2,3}$ Amritsar College of Engineering \& Technology, Amritsar, India
}

\begin{abstract}
Image Segmentation plays a vital role in image processing. The research in this area is still relevant due to its wide applications. Image segmentation is a process of assigning a label to every pixel in an image such that pixels with same label share certain visual characteristics. Sometimes it becomes necessary to calculate the total number of colors from the given RGB image to quantize the image, to detect cancer and brain tumour. The goal of this paper is to provide the best algorithm for image segmentation.
\end{abstract}

Keywords: Image segmentation, $R G B$

\section{INTRODUCTION}

The problem of image segmentation has been known and addressed for the last 30 years. The general description of an image in everybody mind is the list of objects in an image and their positions .But when we deeply examine an image it depicts shadows of an object, differences in the color brightness of an object.[1] Image segmentation is the process of partitioning an image into regions that are in some sense homogeneous, but different from neighboring regions.[2]. Segmentation is the first key step in object recognition, scene understanding and image understanding. Segmentation of an image can be done on the basis of some characteristics such as color, objects that are present in the entire image.[3]. The level to which the separation is carried depends on the problem being solved. [4].The result of image segmentation is a group of different segments that mutually cover the entire image.

Image segmentation algorithms are based on one of the two basic properties of the intensity value i.e discontinuity and similarity. There are different approaches for different type of images. The first approach represents Histogram thresholding, second approach is Edge based and the last one is region based approach. In histogram thresholding different gray or color ranges are represented to made regions of an image . In the second approach, different edge detection operators are used and also the edges are joined if the regions are not connected. In the third approach images are partitioned into regions which are similar according to a set of predefined criteria $[5,6]$.

By concerning the image segmentation as the conundrum of partitioning pixels into different clusters based on color similarity and spacial relation, we propose our color image segmentation method.[7]. It is a segmentation of an image based on colors in it. According to this method, RGB image is firstly converted to hsv image . HSV color space is used because it gives the color according to human perception.

After that the image is converted to $3 \mathrm{rgb}$ planes then the pixels belonging to same color are merged together .After that total numbers of colors are displayed.

This paper is organized in four sections. Section I give an introduction. Steps for proposed algorithm are discussed in section II. Section III discusses the results of the proposed algorithm. Section IV gives Conclusion.

\section{FLOW CHART FOR PROPOSED ALGORITHM}

As the image segmentation is an emerging field in digital image processing in some cases it becomes necessary to find the total number of colors from the original image. It is very useful in many medical applications some of which are detecting brain tumors and cancerous cells. In the proposed method each color present in an image is shown separately. The procedure of calculating total number of colors from an image is very helpful in quantization of image.

In the proposed work the original image which is in RGB is converted to HSV color space .HSV color space is used because it gives color according to human perception After this process image is then converted to $3 \mathrm{RGB}$ planes then the pixels which have same color are merged together. At last the total numbers of colors in an image are calculated. 


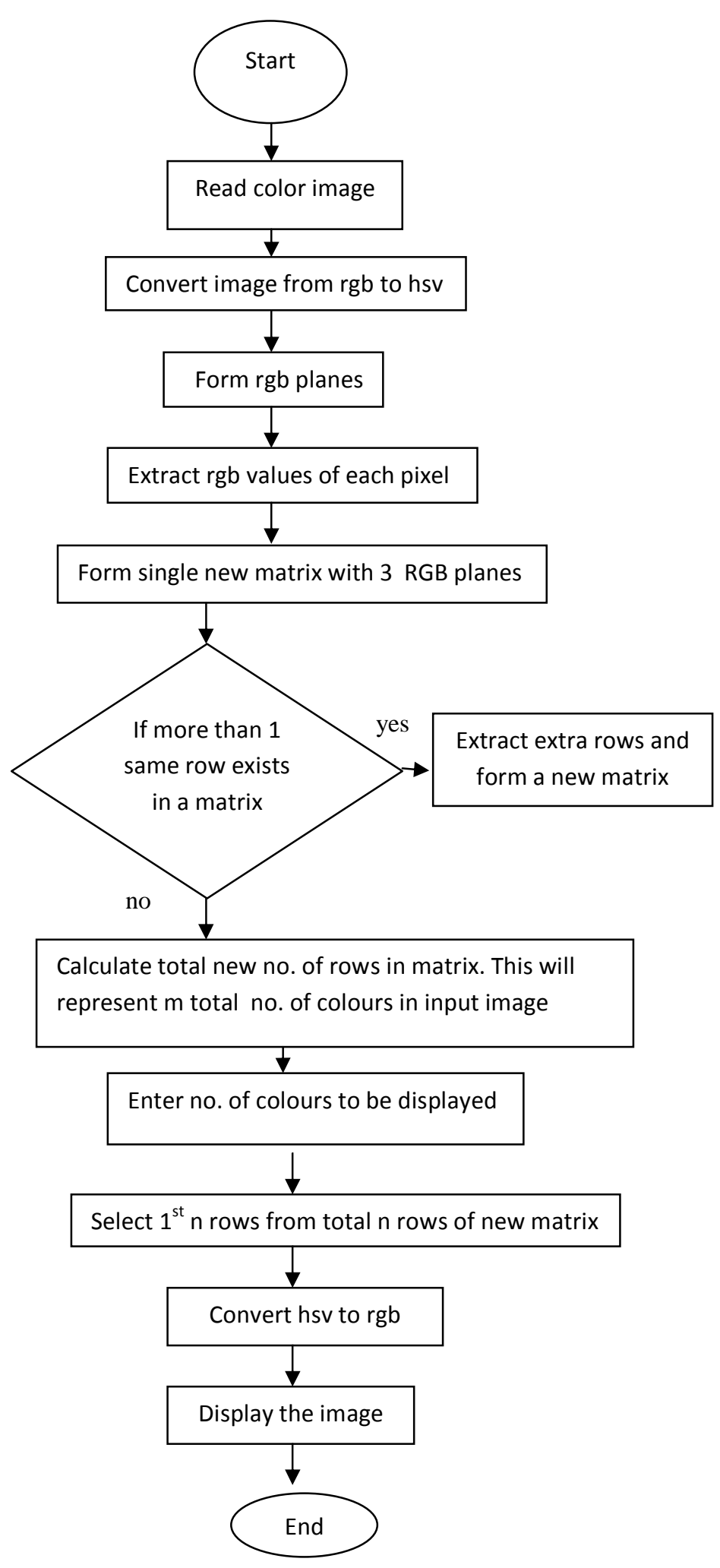

The step wise processes for the proposed algorithm are as follows:

Step 1: The very first step is to read an RGB image

Step 2: The next step is to convert RGB image to HSV. This color space is used because it gives the color according to human perception.

Step3: Further three matrix are made by three different planes.

Step4: A single new matrix is formed so as to see values of RGB at each pixel

Step5: If two rows are equal in a single new matrix then combine those rows

Step6: Total number of colors existing in an original image is calculated

Step7: Enter the number of colors user wants to see

Step8: Image is converted from HSV to RGB color space

\section{RESULTS AND DISCUSSIONS}

We applied our algorithm on various RGB images and as a result we get total number of colors from the given RGB image. The below figure depicts the original RGB image and the other images which are shown below contains the extraction of colors from an original image. The main feature of this algorithm is that, it can calculate the colors in an image automatically which makes the process faster as compare to other algorithms.

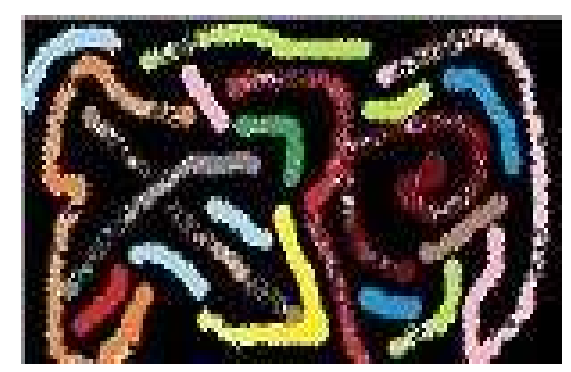

Fig 2: Original image

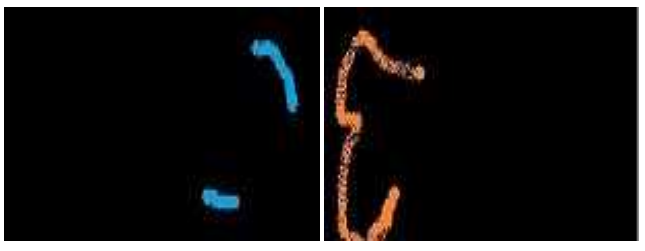

(2a)

(2b)
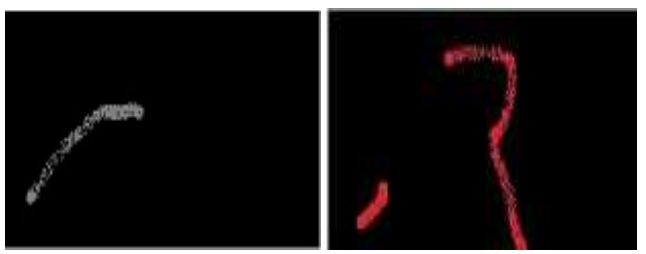

Fig 1: Flow chart for the proposed algorithm

(2c)

(2d) 


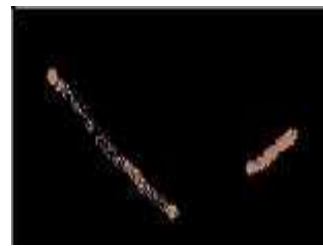

(2e)

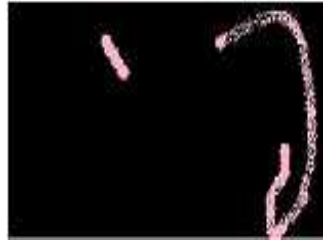

$(2 \mathrm{~g})$

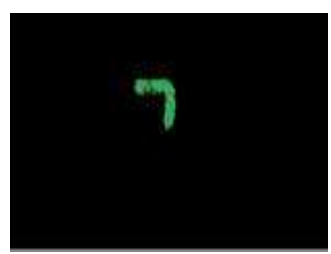

(2i)

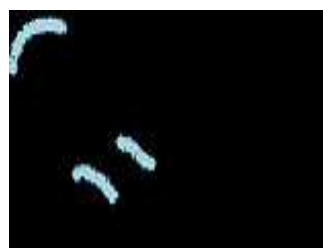

$(2 \mathrm{k})$

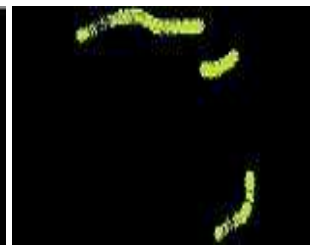

(2f)

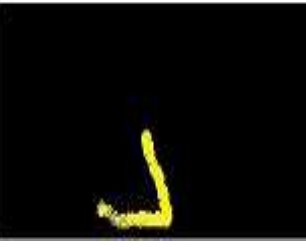

(2h)

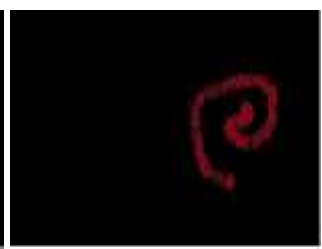

(2j)

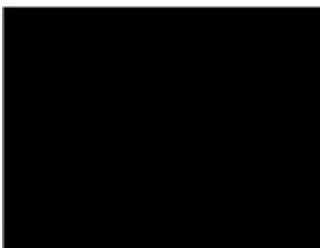

(21)

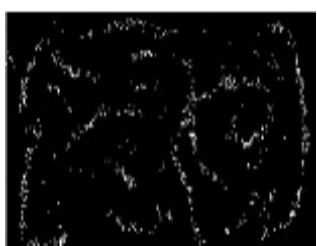

$(2 \mathrm{~m})$

Fig (2a) - (2m) depicts the extraction of each color from an original image.

\section{CONCLUSIONS}

The proposed algorithm follows the image segmentation based on colors at each pixel. This technique is capable because we can get total number of colors from an image automatically which is amazing. If we know the total number of colors then it can be very helpful in medical applications.

\section{REFERENCES}

[1] Gudrun J. Klinker, Steven A. Shafer and Takeo Kanade, "A Physical Approach to Color Image Understanding," International Journal of Computer Vision, Vol. 4, No. 1, pp. 738,1990

[2] Anil Z Chitade "Color based image segmentation using kmeans clustering" International Journal of Engineering science and Technology, Vol. 2(10), 2010, 5319-5325

[3] R. C. Gonzalez and R. E. Woods, Digital Image Processing. Addison-Wesley, 1993

[4] YZang"A review on image segmentation techniques with remote sensing perspective" IAPRS Vienna, Austria, July 5-7, 2010, IAPRS, Vol. XXXVIII

[5] Navneet Kaur et.al " Bacteria foraging based image segmentation "An International Journal of Engineering Sciences ISSN: 2229-6913 Issue July 2012, Vol. 6

[6] Rajeshwar Dass et.al "Image Segmentation Techniques" IJECT Vol. 3, Issue 1, Jan. - March 2012

[7] Y. Zhong, K. Karu et.al, "Locating Text in Complex Color Images," Pattern Recognition, vol. 28, no. 10, pp. 1523-1535, October 1995 